\title{
Life history interactions with environmental conditions in a host-parasite relationship and the parasite's mode of transmission
}

\section{Journal Article}

Author(s):

Agnew, Philip; Koella, Jacob C.

Publication date:

1999

Permanent link:

https://doi.org/10.3929/ethz-b-000422647

Rights / license:

In Copyright - Non-Commercial Use Permitted

Originally published in:

Evolutionary Ecology 13(1), https://doi.org/10.1023/A:1006586131235 


\title{
Life history interactions with environmental conditions in a host-parasite relationship and the parasite's mode of transmission
}

\author{
PHILIP AGNEW* and JACOB C. KOELLA \\ Experimental Ecology, ETH-Zurich, ETH Zentrum NW, CH-8092 Zurich, Switzerland, and \\ Department of Zoology, Aarhus University, Universitetsparken B135, DK-8000 Aarhus C, Denmark \\ (*author for correspondence: Laboratoire d'Ecologie, Université Pierre et Marie Curie (CNRS URA \\ 258), 7 quai St. Bernard, F-75252 Paris, France)
}

\begin{abstract}
The microsporidian parasite Edhazardia aedis is capable of vertical or horizontal transmission among individuals of its host, the mosquito Aedes aegypti, and either mode of transmission may follow the other. We show that following the horizontal infection of host larvae, the parasite's subsequent mode of transmission largely depends on host life history traits and their responses to different environmental conditions. In two experiments the intensity of larval exposure to infection and the amount of food available to them were simultaneously manipulated. One experiment followed the dynamics of host development and the parasite's production of spores while the other estimated the outcome of their relationship. Host life history traits varied widely across treatment conditions while those of the parasite did not. Of particular importance was the host's larval growth rate. Horizontal rather than vertical transmission by the parasite was more likely as low food and high dose conditions favoured slower larval growth rates. This pattern of transmission behaviour with host growth rate can be considered in terms of reproductive value: the potential vertical transmission success that female mosquitoes offer the parasite decreases as larval growth rates slow and makes them more attractive to exploitation for horizontal transmission (requiring host mortality). However, the lack of variation in the parasite's life history traits gave rise in some conditions to low estimates for both its vertical and horizontal transmission success. We suggest that the unresponsive behaviour of the parasite's life history traits reflects a bet-hedging strategy to reduce variance in its overall transmission success in the unpredictable environmental conditions and host larval growth rates that this parasite encounters in nature.
\end{abstract}

Key words: Aedes aegypti, bet-hedging, Edhazardia aedis, microsporidia, mosquito, reproductive value, transmission, virulence

\section{Introduction}

Transmission success is of one of the foremost components of a parasite's fitness. Considerable effort has gone towards developing an understanding of its association with other traits and how their interactions may influence evolution in host-parasite relationships. Among the traits closely linked to transmission success are a parasite's virulence (its cost to host fitness), host resistance, competition for resources within the host, the ecological opportu- 
nities for transmission, and the dynamics of the transmission process itself (e.g. Antia et al., 1994; Bull, 1994; Ewald, 1994; Frank, 1992; Anderson and May, 1991; Hamilton et al., 1990).

The nature of selection arising from the interaction of transmission success with one trait is often made in isolation from its interactions with other traits. Without an appreciation of how various traits interact together it becomes difficult to estimate the importance that selection might have on a particular trait's contribution towards transmission success. Life history theory provides a useful framework with which to integrate various fitness-related parameters and assess their consequences for the evolutionary ecology of host-parasite relationships. For example, life history theory applied to parasites has assessed the role that ecological and demographic parameters can have in shaping the relationship between transmission success and virulence (Frank, 1996), and how selection can favour pleiotropic effects for the timing of virulence in relation to transmission (Michalakis et al., 1992). Selection can also act on host life history traits as a form of resistance against a reduction in their fitness when parasitised (Hochberg et al., 1992; Michalakis and Hochberg, 1994).

Reciprocal interactions among host and parasite life history traits has received less attention so far (Koella et al., 1998). Such interactions are not only expected to occur on an evolutionary time-scale, but have also been shown to be important for each organism's fitness from generation to generation. Effects on the latter scale can be particularly important as the life history traits of many organisms show phenotypic plasticity in different environmental conditions. For example, temperature influences the age at maturity of many organisms. Some parasites manipulate their host's thermal tolerance to favour their own development (Fialho and Schall, 1995), while some hosts exploit temperature conditions to suppress the development of their parasites (Müller and Schmid-Hempel, 1993).

We focus on the role that interactions among a host's and a parasite's life history traits have in determining the parasite's transmission success. In particular, we illustrate interactions between life history traits of the microsporidian parasite Edhazardia aedis and its host the yellow fever mosquito Aedes aegypti in a range of environmental conditions. This host-parasite relationship was chosen for investigation, as E. aedis is capable of vertical and horizontal transmission and either mode of transmission may follow the other (Becnel et al., 1989). The results of two experiments are presented, where larval food availability and exposure to infectious doses of $E$. aedis spores were simultaneously manipulated. One experiment followed the dynamics of host growth and infection while the other recorded the outcome of their relationship. 
The host, the parasite, and their relationship

Aedes aegypti is an important vector for a number of diseases, such as, yellow fever and dengue. It is found throughout the tropics and sub-tropics, where it is particularly associated with human habitation. Extensive details of its ecology, physiology, genetics and behaviour are known (Christophers, 1960). Larvae of Ae. aegypti encounter both spatial and temporal variation in the environmental conditions they experience. Adult female mosquitoes oviposit in a range of natural and artificial containers holding clean freshwater, e.g. bamboo shoots, water storage jars and discarded tyres. These sites typically vary in a number of characteristics, such as temperature, volume, ephemerality and food availability (Focks et al., 1993). This heterogeneity is also experienced among sibs, as female mosquitoes distribute eggs from within a clutch over a number of sites (Apostol et al., 1994).

Edhazardia aedis is an obligate and intracellular microsporidian parasite that is specific to Ae. aegypti (Becnel and Johnson, 1993). Little is known about its natural ecology. The type specimen was described from a single infected mosquito collected in Puerto Rico (Kudo, 1930). It was subsequently re-discovered in Thailand where it was one of the most common pathogens infecting Ae. aegypti larvae (Hembree, 1979). Field trials in North America are currently evaluating its potential as a biological control agent of this mosquito. It has a complex life cycle that involves vertical and horizontal transmission. Furthermore, either mode of transmission may follow the other. An outline of the life cycle and transmission behaviour of $E$. aedis follows; further details can be found in Johnson et al. (1997) and Becnel et al. (1989).

\section{Life cycle and transmission behaviour of Edhazardia aedis}

The typical sequence of transmission behaviour by E. aedis is believed to involve larvae ingesting uninucleate spores from their aquatic environment (Fig. 1). Infection is initiated when these spores germinate and a hollow polar filament extends from within the spore to puncture an epithelial cell in the host's midgut. The spore's contents are directly inoculated into the host cell. The infection then proliferates as $E$. aedis undergoes a series of soft-bodied developmental stages that culminates in the production of binucleate spores. The germination of these spores is believed to be responsible for spreading infection beyond the initial site of infection to other host tissues, particularly to larval oenocyte cells (Johnson et al., 1997). Within oenocyte cells, E. aedis undergoes further development leading to the production of a second type of binucleate spore. These binucleate spores are solely responsible for vertical transmission when they germinate and inoculate a female mosquito's developing oocytes; there is no paternal transmission (Hembree and Ryan, 1982). 


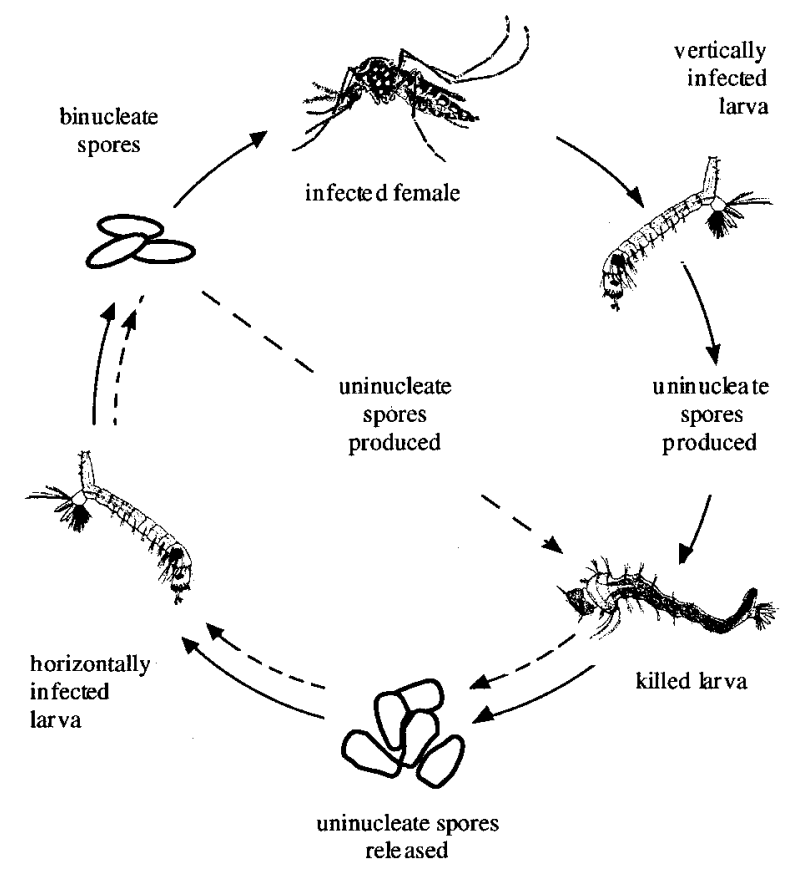

Figure 1. Alternative life cycles of Edhazardia aedis infecting Aedes aegypti. Solid lines indicate the horizontal-vertical transmission sequence. Dashed lines indicate the horizontal-horizontal transmission sequence (see text for details).

Further development and proliferation of E. aedis continues, when infected eggs hatch and larvae develop. Infection in larvae is predominately seen in the host's fat body cells, where uninucleate spore production occurs. Vertically infected larvae in many conditions do not survive beyond pupation: the proliferation of uninucleate spores is associated with their mortality. Following the degradation of the host's cuticle, uninucleate spores are released into the aquatic environment for horizontal transmission to other larvae.

Two deviations from this horizontal-vertical sequence of transmission occur (Becnel et al., 1989).

(i) Following the horizontal infection of a larva E. aedis may complete its whole life cycle within its host before it emerges as an adult. This happens when the second type of binucleate spore germinates and initiates development that would otherwise be seen in the vertically infected generation. If enough uninucleate spores are produced to kill the host before its emergence, these spores will be released into the aquatic environment and be able to infect other larvae. This gives the potential for a horizontal-horizontal sequence of transmission with vertical transmission being bypassed (Fig. 1).

(ii) Alternatively, vertically infected larvae may survive the production of uninucleate spores and emerge as adults. Uninucleate spores that germinate 
within these hosts continue with development as though they had infected another larva via the environment. Consequently, the oocytes of female mosquitoes can be infected by the second type of binucleate spore and a verticalvertical sequence of transmission will be observed with horizontal transmission being bypassed (not shown).

\section{Materials and methods}

The Rockefeller strain of Ae. aegypti from the Swiss Tropical Institute (Basel) was used in both experiments. Edhazardia aedis was provided from laboratory stock maintained at the United States Department of Agriculture (Gainesville, Florida).

\section{Experimental protocols and data collection}

Two experiments manipulated larval food availability and the concentration of uninucleate spores that larvae were exposed to. We were interested in the effects these conditions had on the outcome (Experiment I) and developmental dynamics (Experiment II) of the host-parasite relationship.

In both experiments, several thousand eggs from a large laboratory colony of Ae. aegypti were hatched synchronously under conditions of a partial vacuum. Within six hours of hatching, 20 groups of 200 larvae were separated into plastic rearing trays $(10 \times 10 \times 10 \mathrm{~cm})$ containing $300 \mathrm{ml}$ of tap water. These trays were maintained at $28^{\circ} \mathrm{C}\left( \pm 0.5^{\circ} \mathrm{C}\right)$ and $85 \%$ humidity $( \pm 5 \%)$, with a 12 -h:12-h light:dark cycle on a single shelf in a randomised pattern.

Larval food conditions were manipulated as $25,50,75$, and $100 \%$ of a standard daily ration of ground fish food $\left(\right.$ Tetramin $\left.^{\mathrm{TM}}\right)$. A $100 \%$ ration is just sufficient for this strain of Ae. aegypti to achieve maximum growth rates (unpublished data). The $100 \%$ ration per larva was: day $0,0.06 \mathrm{mg}$, day 1 , $0.08 \mathrm{mg}$, day $2,0.16 \mathrm{mg}$, day $3,0.32 \mathrm{mg}$, and even-numbered days thereafter, $0.64 \mathrm{mg}$.

Larval infections occurred at 72 hours post-hatch in glass beakers containing $100 \mathrm{ml}$ of tap water. Exposure to uninucleate spores lasted for 24 hours. The day's food was also added to the infectious solutions. After exposure to infection, larvae were rinsed in tap water and returned to their original rearing trays that had been refilled with $300 \mathrm{ml}$ of fresh tap water. The range of uninucleate spore concentrations that larvae experienced was $0,10,10^{2}, 10^{3}$, and $10^{4} \mathrm{ml}^{-1}$. These spores were harvested from vertically infected larvae that had been hatched nine days earlier. Approximately 20 larvae were homogenised in a glass tissue grinder in $1 \mathrm{ml}$ of water. Uninucleate spore concentrations were determined with a hemocytometer and light microscope. The spores added to 
infection beakers were serially diluted from the original solution until the desired concentrations were achieved.

Larval and pupal mortality were checked daily. Cadavers were removed and their day of collection and treatment details were recorded before they were individually stored in $1.5 \mathrm{ml}$ plastic vials at $-20^{\circ} \mathrm{C}$ until further investigation. Individuals pupating on a given day from within a treatment were transferred together into pupation chambers consisting of small beakers of water under larger inverted plastic cups. No food was provided for adults and two days after emergence each pupation chamber was frozen. Details of each adult were recorded before they were individually stored at $-20^{\circ} \mathrm{C}$ in $1.5 \mathrm{ml}$ plastic vials. The amount of food provided to larvae in each rearing tray was adjusted daily by subtracting the number of mosquitoes that had been collected due to pupation or mortality from the original treatment size of 200 larvae.

The number of spores produced by E. aedis in mosquitoes exposed to infection was determined by adding $0.5 \mathrm{ml}$ of water to the plastic vials and mechanically homogenising the contents. The resulting solution was re-suspended and the numbers of binucleate and uninucleate spores were counted using a hemocytometer and light microscope. The two types of binucleate spore produced by $E$. aedis cannot practically be distinguished without electron microscopy and so were pooled together as a single population of binucleate spores; this point is addressed in the Discussion. Treatment condition and collection details were unknown to the investigator at the time that spores were counted. Samples of at least 40 individuals from each control treatment $(0$ uninucleate spores $\mathrm{ml}^{-1}$ ) were also checked for spores.

\section{Experiment $I$}

We prepared 20 groups of mosquito larvae as described above. Estimates for the parasite's potential transmission were categorised as follows: control mosquitoes offered no transmission; adult male mosquitoes offered no transmission; adult female mosquitoes exposed to infection and not harbouring spores were judged uninfected and offered no transmission; larvae or pupae dying and harbouring uninucleate spores were classed as capable of horizontal transmission; female mosquitoes emerging with binucleate spores were classified as capable of vertical transmission. This last category was sub-divided into: (i) those females harbouring only binucleate spores, and (ii) those females harbouring binucleate and uninucleate spores. The latter group of females is expected to contribute far less towards vertical transmission due to reduced blood-feeding behaviour (see Discussion, and Koella and Agnew, 1997). In analyses involving transmission, we conservatively assumed these females would offer no vertical transmission success to E. aedis.

A number of mosquitoes died during the period between their emergence and before adult chambers were frozen. Some of these adults were found lying 
on the surface of the water in their pupation beakers while others were found elsewhere in the adult chambers. As a conservative measure we assumed no transmission would result from any of these adults.

Actual spore numbers were not used quantitatively in estimating transmission success as an undue number of assumptions would have been required.

Adult size (wing length) was measured for all female mosquitoes, but is reported only for the uninfected individuals. This is because size does not give a reliable indication of an infected female's fitness, e.g. fecundity (Becnel et al., 1995), and so would not be an informative character for either host or parasite fitness. The fluctuating asymmetry in wing length of female mosquitoes from this experiment is reported elsewhere (Agnew and Koella, 1997).

\section{Experiment II}

This was the same as Experiment I except that from five days post-hatching we collected 10 mosquitoes treatment ${ }^{-1}$ day $^{-1}$ until no more mosquitoes remained in the treatment or adult chambers were frozen. The 10 mosquitoes collected were chosen to reflect the proportions of developmental stages (larvae:pupae:adults) of live mosquitoes within each treatment on that day. The distribution of ages at pupation within a treatment was also incorporated into calculations for the collection of pupae and adults.

\section{Statistical analyses}

The total number of mosquitoes collected from each experiment was less than their original sample sizes. The probability that a mosquito was unaccounted for was assessed by a nominal logistic regression for each experiment. Subsequent analyses are based on the data from collected mosquitoes. Throughout the analyses food availability is taken as a continuous variable and infection dose as an ordinal variable.

Parametric analyses were used when permitted by the data. Non-parametric analyses of variance are based on the ranked averages of response variables (Sokal and Rohlf, 1995).

In Experiment II the number of mosquitoes collected each day was dictated by the experiment's sampling regime and mosquito mortality. However, all the mosquitoes collected on a given day were pooled together and their day of collection was treated as a fixed effect: the majority of mosquitoes were collected while alive and the two populations did not vary systematically in their spore content. Day of collection was analysed as an ordinal parameter as spore production was not linear with respect to the period of collection. This required pooling consecutive days of collection $(5-6,7-8,9-10,11-12)$ to gain sufficient degrees of freedom for the analysis and that data from days 13-14 were excluded to avoid missing cells. 
The statistical package JMP, version 3.1.6, was used for all analyses (SAS Institute, 1994).

\section{Results}

In both experiments, fewer mosquitoes were collected from most treatments than their original sample sizes of 200 larvae (Table 1). In general, the number of mosquitoes collected decreased as larval food availability became less, especially as infectious dose increased (Table 2).

\section{Experiment I}

More mosquitoes were collected due to mortality as larval food availability decreased and infectious dose increased; the effect of dose became greater as food availability decreased (Fig. 2a, Table 3a).

Of the mosquitoes exposed to infection more were classified as potentially capable of transmitting $E$. aedis as infectious dose increased; particularly as larval food availability became less (Fig. 2b, Table 3b). These mosquitoes showed a significant interaction between food and dose treatments for whether this transmission would be vertical or horizontal: less vertical transmission was seen as larval food availability decreased and infectious dose increased (Fig. 2b, Table 3c).

Age at pupation was influenced by treatment conditions (Table 4a). Pupation tended to become later as food availability decreased and as infectious dose increased. Low food and high dose conditions interacted to produce earlier ages at pupation. Essentially the same pattern was seen for ages of preadult mortality (Table 4 b).

Female mosquitoes are pooled across treatment conditions to illustrate the distributions of age at pupation in relation to the parasite's transmission. Figure 3a shows the distributions for control females and females classified as

Table 1. Number of mosquitoes collected in treatment conditions of Experiments I and (Experiment II) from the original 200 larvae

\begin{tabular}{|c|c|c|c|c|c|c|}
\hline \multirow[t]{2}{*}{ Food } & \multicolumn{6}{|l|}{ Dose } \\
\hline & 0 & $10^{1}$ & $10^{2}$ & $10^{3}$ & $10^{4}$ & Mean \\
\hline 25 & 188 (113) & 188 (77) & $182(96)$ & 138 (77) & 156 (107) & 171 (94) \\
\hline 50 & 198 (133) & $188(151)$ & 200 (114) & $200(129)$ & $189(126)$ & $194(131)$ \\
\hline 75 & $194(140)$ & $198(150)$ & 191 (129) & $191(145)$ & 171 (135) & $191(140)$ \\
\hline 100 & 200 (167) & $192(156)$ & 192 (189) & $192(154)$ & 129 (152) & 181 (164) \\
\hline Mean & 195 (138) & $192(134)$ & $193(132)$ & $181(126)$ & $161(130)$ & \\
\hline
\end{tabular}


Table 2. Nominal logistic analyses for the probability of collecting mosquitoes from each experiment

\begin{tabular}{|c|c|c|c|c|c|}
\hline \multirow[t]{2}{*}{ Source } & \multirow[t]{2}{*}{$d f$} & \multicolumn{2}{|c|}{ Experiment I } & \multicolumn{2}{|c|}{ Experiment II } \\
\hline & & Wald $\chi^{2}$ & $p$ & Wald $\chi^{2}$ & $p$ \\
\hline Food & 1 & 11.211 & 0.001 & 33.961 & $<0.001$ \\
\hline Dose & 4 & 47.134 & $<0.001$ & 17.554 & 0.002 \\
\hline Food $\times$ dose & 4 & 96.446 & $<0.001$ & 17.884 & 0.001 \\
\hline
\end{tabular}

uninfected. In Figure $3 b$ the females from both categories of vertical transmission are shown along with the distribution of age at pupation for mosquitoes that died as pupae (both sexes) and were capable of horizontal transmission. Figure 4 shows age at pupation and size at emergence of control female mosquitoes from the four food treatments with estimates of their fecundity based on Briegel (1990).

\section{Experiment II}

There were no significant differences across treatment conditions for the day at which the first $10 \%$ of individuals within a treatment harboured binucleate spores (Table 5). The median time for the appearance of these spores in mosquitoes was on day 7 , or 4 days post-infection. There were not enough mosquitoes within each treatment that harboured uninucleate spores for a similar analysis. However, the first $75 \%$ of mosquitoes harbouring uninucleate spores were collected on days 11-12, with the remaining $25 \%$ collected on days 13-14. These mosquitoes included larvae, pupae and adults. Two larvae that harboured uninucleate spores on days 5 and 6 , respectfully, were excluded from this calculation as the presence of these spores almost certainly reflected residual gut contamination from the time of exposure to infection.

The mean number of binucleate and uninucleate spores found in mosquitoes increased with time since infection and more so as infectious dose increased (Fig. 5, Table 6). More uninucleate spores were also found for a given dose and day of collection as larval food availability decreased (Table 6). Neither spore type was found in the individuals examined from control treatments. This confirms that the results represent actual spore production rather than the possible occurrence of other morphologically similar bodies, such as fungal spores or algae.

The dynamics of mosquito development and mortality changed with treatment condition (Fig. 6, Table 7). Overall, the rate at which mosquitoes entered pupation or emerged as adults was slower as larvae experienced lower food availability and higher infectious doses. The slower rate of development 


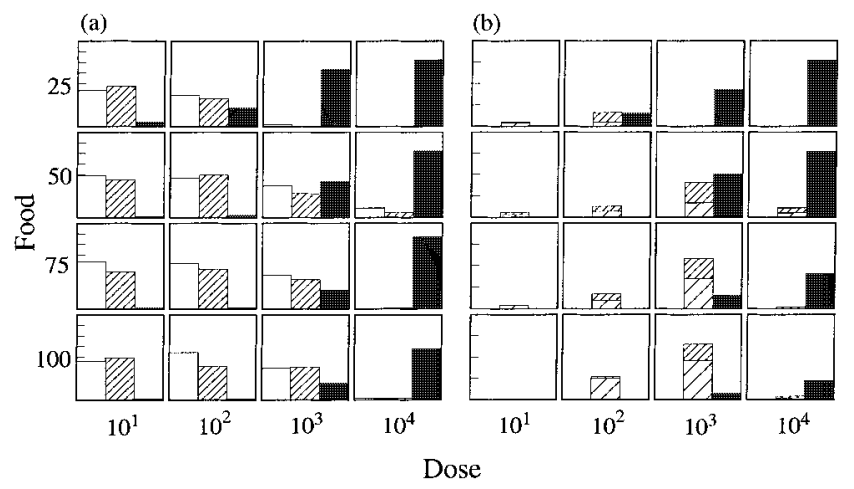

Figure 2. Host survival and potential parasite transmission across the experimental treatment conditions. (a) The open columns are the number of males surviving to adulthood, the hatched columns are the number of females surviving to adulthood, and the solid columns are the number of individuals dying as larvae or pupae. The $y$-axis in each diagram ranges from $0-200$, with increments of 25 shown (b) Hatched columns are the number of adult females capable of transmitting E. aedis vertically to their offspring, where broad hatching is for females harbouring only binucleate spores and fine hatching is for females harbouring both spore types. The solid columns are the number of individuals dying before adulthood and potentially capable of transmitting $E$. aedis horizontally to other larvae. The $y$-axes ranges from $0-100$, increments of 25 are shown.

associated with lower food availability and higher doses also lead to proportionately more mosquitoes being collected as a result of pre-adult mortality; more of these individuals were collected due to larval rather than pupal mortality as dose increased. Furthermore, within treatments there was a pattern in

Table 3. Nominal logistic analyses for host and parasite parameters from Experiment I

\begin{tabular}{lccc}
\hline Source & $d f$ & Wald $\chi^{2}$ & $p$ \\
\hline (a) Probability of mosquitoes being & collected dead or alive & \\
Food & 1 & 7.688 & 0.006 \\
Dose & 4 & 133.947 & $<0.001$ \\
Food $\times$ dose & 4 & 30.096 & $<0.001$ \\
$n=3701$ & & \\
(b) Probability of vertical or horizontal transmission for E. aedis from treatments exposed to \\
infection \\
Food & 1 & \\
Dose & 3 & 0.283 & 0.594 \\
Food $\times$ dose & 3 & 95.902 & $<0.001$ \\
$n=2885$ & & & $<0.001$ \\
(c) Probability of vertical or horizontal transmission from mosquitoes capable of transmission \\
Food & 1 & 0.737 & 0.391 \\
Dose & 3 & 7.289 & 0.063 \\
Food $\times$ dose & 3 & 11.038 & 0.012 \\
$n=495$ & &
\end{tabular}


Table 4. Non-parametric ANOVA for treatment effects on ages of pupation and pre-adult death (days) in Experiment I

\begin{tabular}{lrrr}
\hline Source & $d f$ & \multicolumn{1}{l}{$H$} \\
\hline (a) Age at pupation & & & \multicolumn{1}{l}{} \\
Food & 1 & 895.433 & $<0.001$ \\
Dose & 4 & 6.246 & 0.182 \\
Food $\times$ dose & 4 & 10.931 & 0.027 \\
$n=3275$ & & \\
(b) Age of pre-adult mortality & & & \\
Food & 1 & 11.429 & 0.001 \\
Dose & 4 & 61.046 & $<0.001$ \\
Food $\times$ dose & 4 & 37.196 & $<0.001$ \\
$n=983$ & & & \\
\hline
\end{tabular}

the timing of mortality; larval death tended to make an increasing contribution towards pre-adult mortality as day of collection became later.

\section{Discussion}

Simultaneously manipulating food availability for Ae. aegypti larvae and their exposure to infectious doses of $E$. aedis produced strong interactions for several

(a)

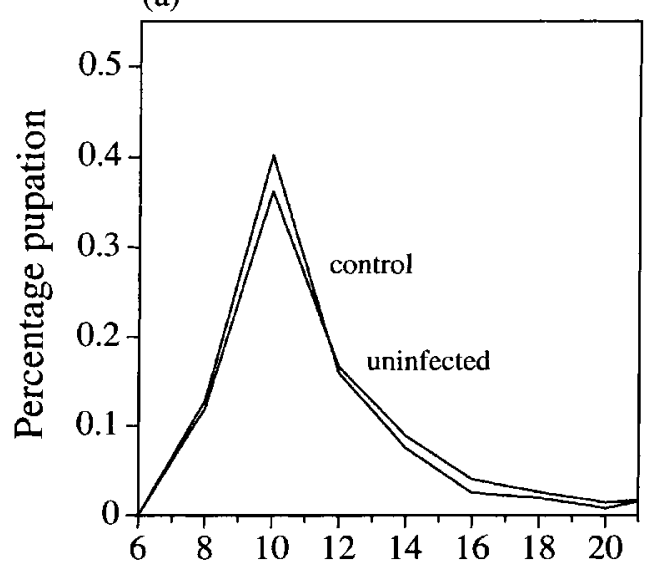

(b)

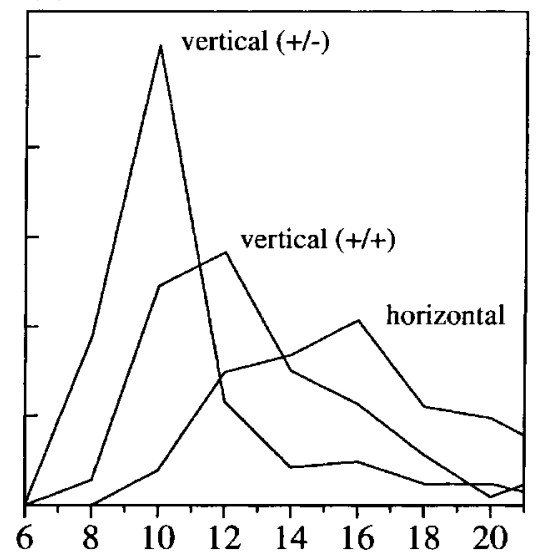

Age at pupation (days)

Figure 3. Distributions for ages at pupation for (a) pooled control females and females classified as uninfected, (b) ages of pupation for individuals capable of transmitting E. aedis; vertical (+/ -$)$ are females harbouring only binucleate spores, vertical $(+/+)$ are females with binucleate and uninucleate spores, and horizontal are individuals that died during pupation and harboured uninucleate spores. 


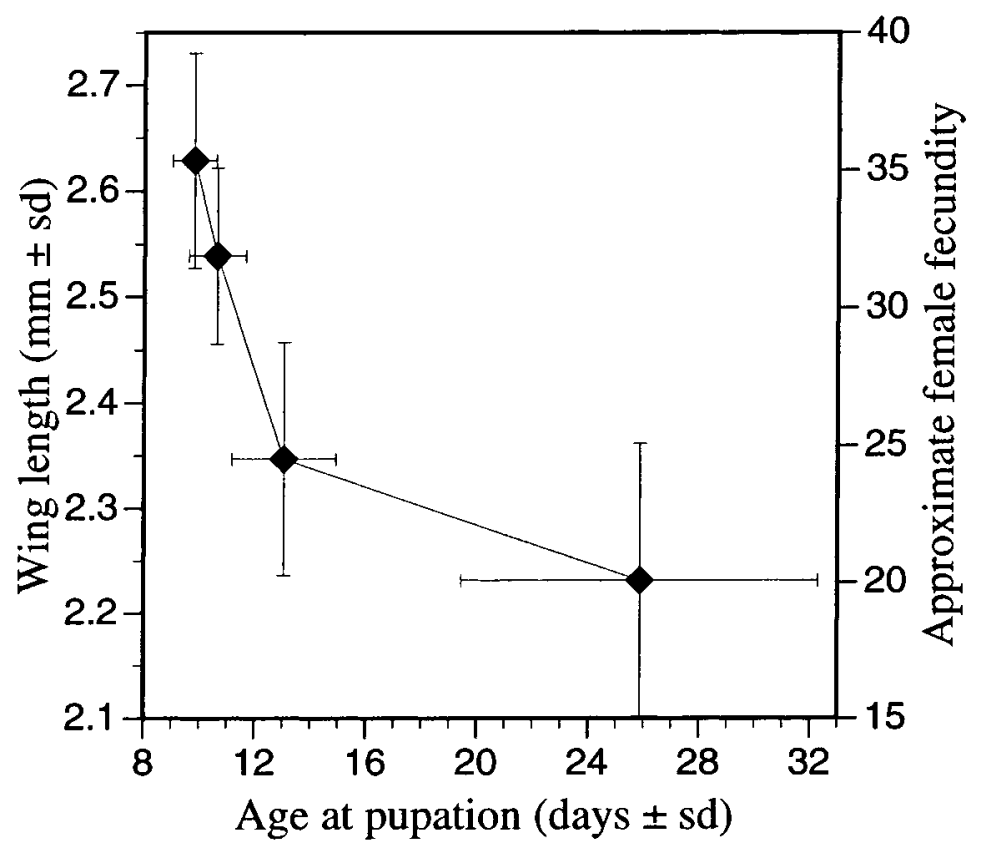

Figure 4. Age and size of control female mosquitoes from each food treatment with an estimate of their fecundity. Food treatments from left to right: 100, 75, 50 and 25\% of standard diet.

fitness-related parameters in the host-parasite relationship. These interactions arose from within single generations of relatively homogenous genetic stock and were largely governed by variation in host rather than parasite life history traits. Before turning to these interactions, the number of mosquitoes accounted for in each experiment is addressed.

\section{Missing mosquitoes}

The number of mosquitoes collected from each experiment was less than their original sample sizes (Table 1). In both experiments, there was a general trend

Table 5. ANOVA for the day that the first $10 \%$ of binucleate spore harbouring individuals were collected within exposed treatments of Experiment II

\begin{tabular}{lrrll}
\hline Source & $d f$ & \multicolumn{1}{l}{ ss } & \multicolumn{1}{l}{$F$} & $p$ \\
\hline Food & 1 & 0.061 & 0.073 & 0.790 \\
Dose & 3 & 2.158 & 0.860 & 0.472 \\
Food x dose & 3 & 2.430 & 0.969 & 0.420 \\
Error & 31 & 25.926 & & \\
$r^{2}=0.19$ & & & & \\
\hline
\end{tabular}




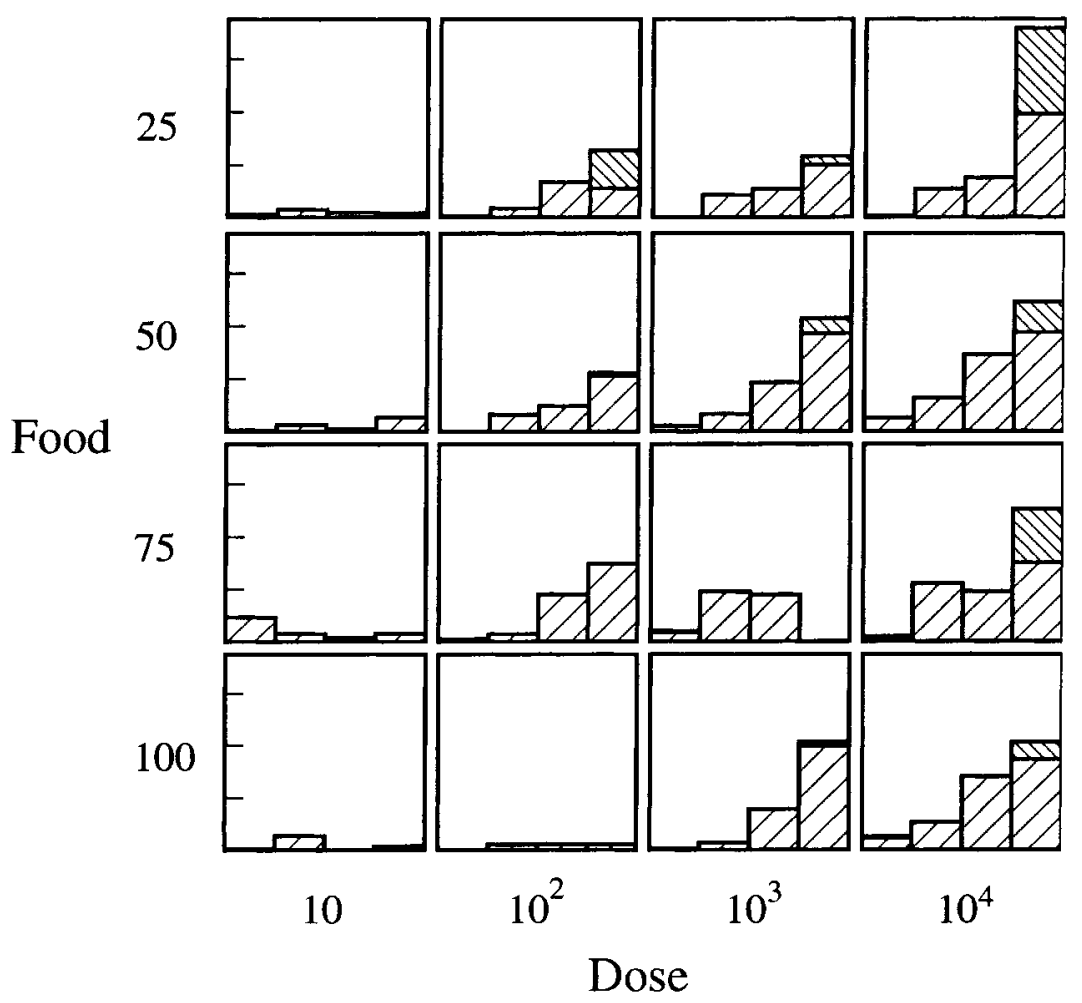

Figure 5. Mean spore production of E. aedis over time and across treatment conditions. The $y$-axis is common in each graph and increases in units of 2000 spores host $^{-1}$. The four columns along the $x$-axis within each graph indicate production on days 5-6, 7-8, 9-10, and 11-12, respectfully. No data were collected on days $11-12$ from the $75 \%$ food level and dose $10^{3}$. The broadly hatched areas sloping up to the right are for binucleate spore number, with uninucleate spore number in the finely hatched sloping up to the left.

Table 6. Non-parametric ANOVA for spore production among treatments over the collection period in Experiment II

\begin{tabular}{|c|c|c|c|c|c|}
\hline \multirow[t]{2}{*}{ Source } & \multirow[t]{2}{*}{$d f$} & \multicolumn{2}{|c|}{ Binucleate } & \multicolumn{2}{|c|}{ Uninucleate } \\
\hline & & $H$ & $p$ & $H$ & $p$ \\
\hline Food & 1 & 0.058 & 0.810 & 0.000 & 1.000 \\
\hline Dose & 3 & 0.286 & 0.963 & 0.173 & 0.982 \\
\hline Food $\times$ dose & 3 & 2.174 & 0.537 & 0.473 & 0.925 \\
\hline Day & 3 & 0.613 & 0.893 & 1.260 & 0.739 \\
\hline Food $\times$ day & 3 & 0.196 & 0.978 & 0.896 & 0.826 \\
\hline Dose $\times$ day & 9 & 24.473 & 0.004 & 80.436 & $<0.001$ \\
\hline $\begin{array}{l}\text { Food } \times \text { dose } \times \text { day } \\
n=1159\end{array}$ & 9 & 10.550 & 0.308 & 34.624 & $<0.001$ \\
\hline
\end{tabular}




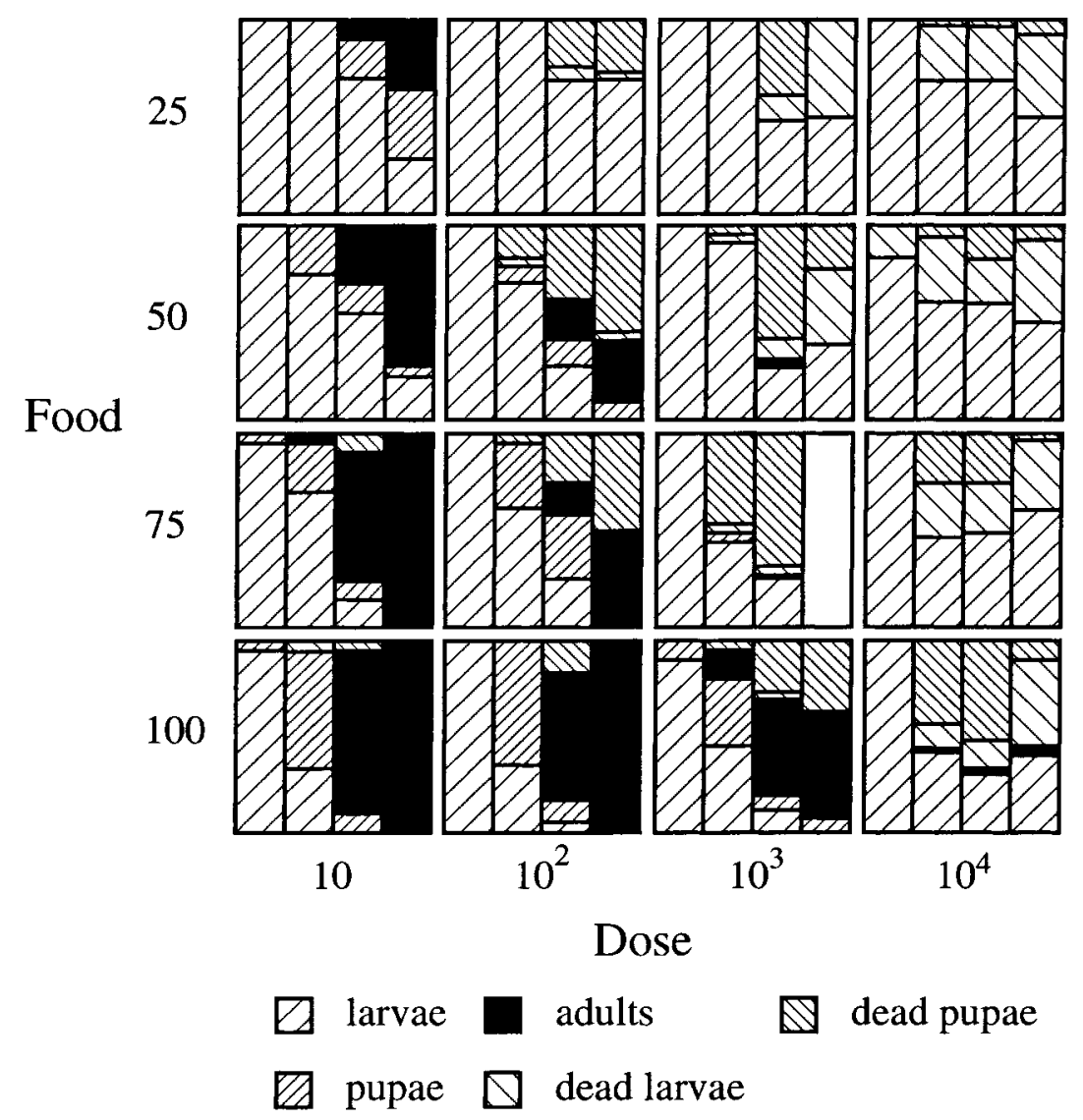

Figure 6. Distributions of Ae. aegypti developmental stages collected over time and across treatment conditions. The $x$-axis within each graph represents time of collection as in Fig. 5. No data were collected on days $11-12$ from the $75 \%$ food level and dose $10^{3}$. The $y$-axis is common in each graph and represents the sum of collection divided up into the percentages that each developmental stage contributed.

for fewer mosquitoes to be collected as larval food availability decreased and as dosage increased (Table 2). We suspect the majority of unaccounted or 'missing' mosquitoes can be attributed to larvae cannibalising one another, particularly as cannibalism was noticed during both experiments (Agnew, personal observation). This form of interaction is of interest as it has consequences for the fitness of each organism and the potential for transmission of infection among hosts.

Competitive interactions among larvae are regarded as the major densitydependent factor in the life history of Ae. aegypti, and are especially intense for early larval instars (Dye, 1984). Larval competition increases as food availability decreases (Southwood et al., 1972; Gilpin and McClelland, 1979), and 
Table 7. Nominal logistic analysis for treatment conditions and day of collection on the developmental stages of the collected mosquitoes in Experiment II

\begin{tabular}{lccl}
\hline Source & $d f$ & Wald $\chi^{2}$ & $p$ \\
\hline Food & 4 & 0.833 & 0.934 \\
Dose & 4 & 0.432 & 0.980 \\
Food $\times$ dose & 4 & 0.458 & 0.977 \\
Day & 16 & 27.601 & 0.035 \\
Food $\times$ day & 16 & 37.391 & 0.002 \\
Dose $\times$ day & 16 & 9.705 & 0.882 \\
Food $\times$ dose $\times$ day & 16 & 26.276 & 0.050 \\
$n=2076$ & & \\
\hline
\end{tabular}

larger larvae sometimes cannibalise smaller larvae as a source of food (MacGregor, 1915). The greater number of missing mosquitoes in both experiments as larval food availability decreased fits these earlier observations.

The additional role of parasitism in modifying competitive interactions among Ae. aegypti larvae does not appear to have been addressed previously. The effect of infection could reduce the competitive ability of larvae or slow their growth rates. Greater competitive ability will promote earlier and faster larval growth. In turn, as larvae become bigger they filter more water and are more efficient at assimilating energy from their diet (Gilpin and McClelland, 1979). Consequently, initial differences in competitive ability among larvae may be amplified by costs associated with infection and reinforced with time.

The effect of E. aedis on larvae of Ae. aegypti need not necessarily involve actual infection. Guts of dipteran larvae are sometimes blocked by indigestible microsporidian spores (Cossentine and Lewis, 1986). Such blockages result in death of these larvae by preventing the further intake and assimilation of food. This could have occurred in larvae of Ae. aegypti, particularly as dosages increased. Smaller, less well-fed larvae are probably at more risk of blocked guts than larger larvae ingesting a similar number of spores. Thus, food and dose conditions could interact to promote the opportunity for cannibalism without actually involving infection.

In this host-parasite relationship, it is clear that being the cannibal or cannibalee makes a difference to a mosquito's fitness. Cannibalism can also act on the parasite's fitness by influencing its transmission success. Horizontal transmission is likely to occur if the larvae being consumed is harbouring uninucleate spores. For E. aedis, this may be a more efficient path for horizontal transmission than dispersal via the environment, as fewer spores need to be produced: the concentration of spores within the host will be greater than outside the host. Thus, the density-dependent process of horizontal transmission needs to be considered in two environments: the host and the external environment. Cannibalism can also result in no transmission success for $E$. aedis 
if it has not yet produced uninucleate spores in the larvae being consumed. This will presumably have been the general case in Experiments I and II as only infected larvae over 11 days old harboured uninucleate spores (Fig. 5) and competitive interactions are more intense for young larvae (Dye, 1984). In nature the heterogeneity of larval ages within a site may be much greater than in our experiments. This should influence the relative gains and losses of horizontal transmission success for $E$. aedis in association with cannibalism.

A closer examination of the interplay between competition and infection in host-parasite relationships, particularly considering density-dependent processes and cannibalism, would be useful. This line of discussion cannot be furthered here as not enough data are available. Consequently, we acknowledge such factors may be acting in this relationship but now focus on data from mosquitoes that were collected and where interactions between host and parasite can be inferred more directly.

\section{Experiments I and II}

\section{Parasite life history traits}

The production of binucleate spores by $E$. aedis began around 4 days postexposure, in mosquitoes aged 7-8 days, and did not vary systematically across the treatment conditions of Experiment II (Table 5). This extends the single treatment results of Johnson et al. (1997), where the first type of binucleate spore was also found at 4 days post-exposure. They additionally report that the second type of binucleate spores appeared from 5 days post-exposure; uninucleate spore production is not mentioned. In Experiment II, uninucleate spores were found from 8 days post-exposure in mosquitoes aged 11 days or more. The ability of $E$. aedis to complete its life cycle within a single larva reveals that its own development is not explicitly linked to that of its host. Our results further suggest that $E$. aedis does not vary the rate of progress through its life cycle in response to different food and dosage conditions its host experiences.

Although the initiation of spore production did not vary in Experiment II, the number of binucleate and uninucleate spores subsequently produced did; more spores were found as infectious dosages increased (Table 6). This pattern is likely to emerge from a property of the infection as a whole rather than differential behaviour of $E$. aedis itself: increasing the intensity of exposure to microsporidian spores increases both the probability that a host will be infected and the number of spores that establish the infection (e.g. Ebert, 1995). This would also suggest that the initiation of spore production by E. aedis is not influenced by competition among different lineages of infection within the host.

Following their appearance, the number of binucleate spores harboured by mosquitoes continued to accumulate with time (Table 6). The two types of 
binucleate spore produced by $E$. aedis are pooled together in this analysis, but no stepwise increase in spore numbers was apparent to indicate that $E$. aedis underwent two sequential and concentrated bouts of binucleate spore production (Fig. 5). As spores themselves cannot replicate, the data suggests that $E$. aedis invested some of its resources into spore production, while also maintaining the proliferation of pre-spore developmental stages. Constraints imposed by host life history traits may favour this form of development (Koella and Antia, 1995). The first type of binucleate spore must be produced in time to spread infection beyond midgut tissues before they degenerate and are shed at the time of pupation (Johnson et al., 1997). If the second and vertically transmitting type of binucleate spore is produced too late, the opportunity to infect a female's first clutch of ripening eggs may be missed. Even under laboratory conditions the proportion of Ae. aegypti females that survive beyond their first oviposition is limited (Christophers, 1960) and will presumably be less in nature. If the time between infection and these host life history events varies unpredictably, an early but partial investment into spore production by E. aedis can help to reduce the risk of producing spores too late, while also allowing the continued production of spores to increase the potential for infection to spread to other tissues or developing oocytes.

A similar argument to the above can be applied to the pattern of uninucleate spore production. An additional interaction was seen with larval food conditions (Table 6); more uninucleate spores were found for a given dosage on a given day as food availability decreased. This pattern could emerge from an increased investment into uninucleate spore production. Alternatively, larvae may provide a better environment for uninucleate spore production than pupae or adults: larvae made greater contributions to the mosquitoes collected as food availability became less (Fig. 6).

\section{Host life history traits}

In contrast to $E$. aedis, the development of $A e$. aegypti varied considerably across treatment conditions in both experiments. As larval food availability became less, female mosquitoes from the control treatments pupated later and emerged as smaller adults (Fig. 4). The observed reaction norm agrees well with predictions from life history theory for organisms with determinate growth that are assumed to be maximising their reproductive success as juvenile growth slows (Berrigan and Koella, 1994).

The presence of an E. aedis infection can be expected to have some negative effect on its host's growth rate. This could be due to energetic costs associated with the proliferation of infection or from the rupturing of host cells with spore production. Such costs are expected to be greater as larvae experienced higher dosage conditions and more uninucleate spores established the initial infections. Mosquitoes did tend to pupate later in higher dosage conditions and as 
food availability became less (Table 4a). However, the age at pupation in the lowest food and highest dosage treatments was earlier than expected from the effects that food and dose treatments had by themselves (Table 4a). This interaction can be considered as a consequence of infections killing their hosts as larvae. To enter pupation (when feeding is not possible) a mosquito larva needs to have a sufficient amount of stored energetic reserves (Gilpin and McClelland, 1979), otherwise the larval period can be prolonged indefinitely (Christophers, 1960). To satisfy this physiological constraint, uninfected larvae can continue to feed and build up their reserves. Infected larvae on the other hand also have to contend with the mounting costs of a developing infection. Furthermore, as a larva's growth rate slows it provides a longer period for the costs of an infection to accumulate and further slow growth. Such a developmental feedback will continue to slow larval growth rates until they either pupate or the mounting costs of infection exhaust hosts and they die. Overall, conditions for larval growth were least favourable in the lowest food and highest dose treatments. However, low food conditions also promote competition among larvae and increase variance in their growth rates (c.f. means and errors for age at pupation in Figure 4). As discussed above, we expect the presence of infection to have a similar effect. Thus, the mean larval growth rate in low food and high dose conditions will have been slow and resulted in most mosquitoes being killed by infection before reaching adulthood and particularly as larvae (Figs 2a and 6; Tables 3a and 7). The growth rates of larvae in the tails of the distribution of growth rates will have either been (i) particularly slow; resulting in early mortality (Table $4 b$ ), or (ii) particularly fast; enabling a few larvae to satisfy criteria for pupation at an early age (Table 4a). Thus, the observed interaction for earlier ages at pupation can be explained by the increased larval mortality that was associated with slower growth rates.

As time since exposure increased there was a general pattern of greater larval rather than pupal mortality (Fig. 6). By not entering pupation, infected mosquitoes could continue to feed as larvae and thereby prolong their survival relative to those pupating.

\section{Patterns of transmission}

Interactions among the life history traits of host and parasite, as shaped by the environmental conditions they experienced, were influential in determining the parasite's transmission behaviour.

In low dosage conditions, many female mosquitoes emerged without harbouring binucleate spores and were classified as uninfected (compare Figs 2a and $2 b$ ). The distribution of ages at pupation for these uninfected female mosquitoes was very similar to that of control female mosquitoes (Fig. 3a). Furthermore, adult mosquitoes were only collected 2 days after their emergence and so approximately 4 days after they entered pupation. Thus, even the 
first females to pupate on day 6 in the uninfected population should have shown binucleate spore presence by the time of their collection (day 10) if they had been infected.

As larvae experienced increased exposure to infection they were more likely to offer E. aedis some form of transmission success, more so as food availability decreased (Table $3 b$ ). The probability that this transmission would be horizontal rather than vertical increased with higher dosage and lower food treatments (Table 3c). To interpret these patterns it helps to bear in mind the variation in Ae. aegypti life history traits relative to those of $E$. aedis. Where exposure to infection was not too intense, female larvae in good food conditions had sufficiently fast growth rates to reach pupation and emerge as adults by the time E. aedis had only produced binucleate spores. As conditions favoured slower larval growth rates and later pupation, some female mosquitoes were still able to enter and emerge from pupation but were more likely to be harbouring both binucleate and uninucleate spores. We classified these females separately as the presence of uninucleate spores represents a substantial cost to the potential vertical transmission success of $E$. aedis: The probability of blood feeding and the volume that a blood meal is drops dramatically in the presence and with the number of uninucleate spores while the presence and number of binucleate spores has no effect on feeding behaviour (Koella and Agnew, 1997). As age at pupation continued to get later mosquitoes were increasingly likely to die as pupae and harbour uninucleate spores, thus making them capable of contributing towards the repeated pattern of horizontal transmission. This transition in transmission behaviour with later ages at pupation is summarised in Fig. 3b. Mosquitoes that died as larvae and harboured uninucleate spores could also contribute towards repeated horizontal transmission. The timing of larval mortality tended to be later than that for pupae (Fig. 6) and thus provided $E$. aedis with more time to produce uninucleate spores.

The interaction for increased overall transmission success of $E$. aedis in treatments where horizontal transmission dominated (Tables $3 b, c)$ is enhanced by the ability of both sexes of Ae. aegypti to contribute towards horizontal transmission while only female mosquitoes can offer vertical transmission.

Rather than manipulating larval growth rates with differential food availability we have also selected mosquitoes for either early or late ages at pupation. The results of each approach are equivalent: as larval growth rates slow, a bias away from vertical and towards horizontal transmission is seen (Koella and Agnew, 1999).

Transmission patterns in an evolutionary context

Variation in host rather than parasite traits largely determined the pattern of events observed in this host-parasite relationship. The apparent lack of any functional response in the life history traits of E. aedis sometimes resulted in 
female mosquitoes emerging with uninucleate spores and being unlikely to offer any transmission success. This situation was particularly common in some treatment conditions (Fig. 2b) and represented approximately 40\% (106/270) of the female mosquitoes capable of vertical transmission in Experiment I.

It could be that this costly situation arises because the life history traits of $E$. aedis are constrained in their ability to respond to the conditions they experience. However, from a comparative perspective this seems unlikely: related mosquito-infecting microsporidia with vertical and horizontal transmission specifically germinate their vertically transmitting spores in response to cues that their female hosts have blood fed (Lord and Hall, 1983). Such conditional germination behaviour would not only minimise any costs to a female mosquito's reproductive success that are associated with the parasite's development and production of horizontally transmitting spores (Agnew and Koella, 1997; Koella and Agnew, 1997) but may also increase vertical transmission success by enhancing the probability of infecting a female's developing oocytes (Lucarotti and Andreadis, 1995).

To understand why the life history traits of $E$. aedis might be as they appear, it is worth considering the parasite's transmission success over a number of generations rather than from a single generation, as in the experimental data. This is because $E$. aedis experiences considerable temporal variation both within and between generations of its host. Therefore, the appropriate measure of fitness to consider is its mean geometric fitness; this parameter is maximised by minimising variation in fitness over generations (Gillespie, 1974).

Now consider the potential fitness that $E$. aedis can gain from female mosquitoes with vertical transmission. The adult size of a female Ae aegypti mosquito is positively correlated with her fecundity and adult longevity (Briegel, 1990). Slower larval growth rates lead to later ages of pupation and smaller adult sizes (Fig. 4). Consequently, the potential reproductive success of female Ae. aegypti and the potential vertical transmission success they offer $E$. aedis will decrease as larval growth rates slow. If host larvae are growing slowly, then E. aedis may do better to exploit them for uninucleate spore production and horizontal transmission. This type of behaviour fits well with the concept of reproductive value (Fisher, 1958) in its application to hostparasite relationships (Frank, 1996). Essentially, the parasite's allocation of resources towards vertical or horizontal transmission can be viewed in terms of each pathway's potential contribution towards the future generations of the parasite. In this host-parasite relationship, the reproductive value of horizontal transmission should increase relative to that of vertical transmission as conditions favour slower larval growth rates. Consequently, the relatively fixed life history traits of $E$. aedis seem geared to providing it with at least some transmission success across the unpredictable range of environmental condi- 
tions and larval growth rates it encounters in nature. Such behaviour seems likely to reduce variance in its overall transmission success across a number of generations than if it stuck rigidly to a horizontal-vertical sequence of transmission. Equally, an adherence to repeated horizontal transmission without the benefit of dispersal associated with vertical transmission would be risky due to the ephemeral nature of larval habitats, particularly as the viability of uninucleate spores in the environment is limited to days rather than weeks (Undeen and Becnel, 1992).

The developmental behaviour shown by E. aedis also fits well with the description of a bet-hedging strategy (Seger and Brockman, 1987): some expected fitness (vertical transmission) is sacrificed for a reduction in the variance of fitness (overall transmission) across a number of generations in a temporally varying environment. A bet-hedging strategy has also been implied for the developmental behaviour of the ciliate protozoan Lambornella clarki in its relationship with Aedes sierrensis; another container-inhabiting mosquito (Philippi and Seger, 1989).

\section{Conclusion}

The effects of simultaneously manipulating (only) two environmental parameters, larval food availability and their intensity of exposure to infection, produced a number of interactions that influenced fitness-related traits for both organisms in this host-parasite relationship. Of particular importance was the variation generated in host life history traits relative to those of the parasite. More specifically, where larvae experienced manageable burdens of infection and sufficient food availability to allow the pupation and emergence of female mosquitoes with relatively high reproductive potentials, the probability of vertical transmission was highest. As food and infection conditions combined to reduce the potential reproductive success of female mosquitoes, the probability of horizontal transmission became greater. This relative contribution of vertical or horizontal transmission success to the parasite's fitness as a function of host life history traits fits well with Fisher's concept of reproductive value. The general unresponsiveness of the parasite's life history traits in our experiments is predicted to reduce the variance of its overall transmission success in the unpredictable environmental conditions it encounters in nature from generation to generation and can be considered as a bet-hedging strategy.

We hope to have illustrated the potential importance that environmental heterogeneity can have on a host-parasite relationship(s) and the utility of life history theory in linking together different traits and their contribution towards a parasite's net fitness. 


\section{Acknowledgements}

We thank W. Rudin and J. J. Becnel, respectively, for supplying mosquito and parasite materials. We also thank F. B. Christiansen, Y. Michalakis and anonymous reviewers for constructive comments on earlier manuscripts and R. Holdgaard for help with our Engilsh.

\section{References}

Agnew, P. and Koella, J.C. (1997) Virulence, parasite mode of transmission, and host fluctuating asymmetry. Proc. R. Soc. Lond. B 264, 9-15.

Anderson, R.M. and May, R.M. (1991) Infectious diseases of humans: dynamics and control. Oxford University Press, Oxford.

Antia, R., Levin, B.R. and May, R.M. (1994) Within-host population dynamics and the evolution and maintenance of microparasite virulence. Am. Nat. 144, 457-472.

Apostol, B.L., Black, W.C.I., Reiter, P. and Miller, B.R. (1994) Use of randomly amplified polymorphic DNA amplified by polymerase chain reaction markers to estimate the number of Aedes aegypti families at oviposition sites in San Juan, Puerto Rico. Am. J. Trop. Med. Hyg. 51, 89-97.

Becnel, J.J., Garcia, J.J. and Johnson, M.A. (1995) Edhazardia aedis (Microspora: Culicosporidae) effects on the reproductive capacity of Aedes aegypti (Diptera: Culicidae). J. Med. Entomol. 32, $549-553$.

Becnel, J.J. and Johnson, M.A. (1993) Mosquito host range and specificity of Edhazardia aedis (Microspora, Culicosporidae). J. Am. Mosq. Control Assoc. 9, 269-274.

Becnel, J.J., Sprague, V., Fukuda, T. and Hazard, E.I. (1989) Development of Edhazardia aedis (Kudo, 1930) N.G., N. Comb. (Microsporida: Amblyosporidae) in the mosquito Aedes aegypti (L.) (Diptera: Culicidae). J. Protozool. 36, 119-130.

Berrigan, D. and Koella, J.C. (1994) The evolution of reaction norms: simple models for age and size at maturity. J. evol. Biol. 7, 549-566.

Briegel, H. (1990) Metabolic relationship between female body size, reserves, and fecundity of Aedes aegypti. J. Insect Physiol. 36, 165-172.

Bull, J.J. (1994) Perspective: Virulence. Evolution 48, 1423-1437.

Christophers, S.R. (1960) Aedes aegypti (L.) the yellow fever mosquito. Its life history, bionomics and structure. Cambridge University Press, Cambridge.

Cossentine, J.E. and Lewis, L.C. (1986) Impact of Vairimorpha necatrix and Vairimorpha sp. (Microspora: Microsporida) on Bonnetia comta (Diptera: Tachinidae) within Agrotis ipsilon (Lepidoptera: Noctuidae) hosts. J. Invertebr. Pathol. 47, 303-309.

Dye, C. (1984) Competition amongst larval Aedes aegypti: the role of interference. Ecol. Entomol. 9, 355-357.

Ebert, D. (1995) The ecological interaction between a microsporidian parasite and its host Daphnia magna. J. Anim. Ecol. 64, 361-369.

Ewald, P.W. (1994) Evolution of Infectious Disease. Oxford University Press, New York.

Fialho, R.F. and Schall, J.J. (1995) Thermal ecology of a malarial parasite and its insect vector: consquences for the parasite's transmission success. J. Anim. Ecol. 64, 553-562.

Fisher, R.A. (1958) The genetical theory of natural selection. Dover Publications, Inc., New York.

Focks, D.A., Haile, D.G., Daniels, E. and Mount, G.A. (1993) Dynamic life table model for Aedes aegypti (L.) (Diptera: Culicidae). Analysis of the literature and model development. J. Med. Entomol. 30, 1003-1017.

Frank, S.A. (1992) A kin selection model for the evolution of virulence. Proc. R. Soc. Lond. B 250, 195-197. 
Frank, S.A. (1996) Models of parasite virulence. Q. Rev. Biol. 71, 37-78.

Gillespie, J.H. (1974) Natural selection for within-generation variance in offspring number. Genetics 76, 601-606.

Gilpin, M.E. and McClelland, G.A.H. (1979) Systems analysis of the Yellow Fever mosquito Aedes aegypti. Fortschr. Zool. 25, 355-388.

Hamilton, W.D., Axelrod, A. and Tanese, R. (1990) Sexual reproduction as an adaptation to resist parasites (a review). Proc. Natl. Acad. Sci. USA 87, 3566-3573.

Hembree, S.C. (1979) Preliminary report of some mosquito pathogens in Thailand. Mosq. News 39, $575-582$.

Hembree, S.C. and Ryan, J.R. (1982) Obervations on the vertical transmission of a new microsporidian pathogen of Aedes aegypti from Thailand. Mosq. News 42, 49-55.

Hochberg, M.E., Michalakis, Y. and de Meeus, T. (1992) Parasitism as a constraint on the rate of life-history evolution. J. Evol. Biol. 5, 491-504.

Johnson, M.A., Becnel, J.J. and Undeen, A.H. (1997) A new sporulation sequence in Edhazardia aedis (Microsporidia: Culicosporidiae), a parasite of the mosquito Aedes aegypti (Diptera: Culicidae). J. Invertebr. Pathol. 70, 69-75.

Koella, J.C. and Agnew, P. (1997) Blood-feeding success of the mosquito Aedes aegypti depends on the transmission route of its parasite Edhazardia aedis. Oikos 78, 311-316.

Koella, J.C. and Agnew, P. (1999) A correlated response of a parasite's virulence and life cycle to selection on its host's life history. J. Evol. Biol. 12, 70-79.

Koella, J.C., Agnew, P. and Michalakis, Y. (1998) Coevolutionary interactions between host life histories and parasite life cycles. Parasitology 116, S47-S55.

Koella, J.C. and Antia, R. (1995) Optimal pattern of replication and transmission for parasites with two stages in their life cycle. Theor. Popul. Biol. 47, 277-291.

Kudo, R. (1930) Studies on microsporidia parasitic in mosquitoes. VIII. On a microsporidian, Nosema aedis nov. sp., parasitic in the larva of Aedes aegypti of Puerto Rico. Arch Protistenkd 69 , $23-28$

Lord, J.C. and Hall, D.W. (1983) Sporulation of Amblyospora (Microsporidia) in female Culex salinarius: Induction by 20-hydroxyecdysone. Parasitology 87, 377-383.

Lucarotti, C.J. and Andreadis, T.G. (1995) Reproductive strategies and adaptations for survival among obligatory microsporidian and fungal parasites of mosquitoes: a comparative analysis of Amblyospora and Coelomomyces. J. Am. Mosq. Control Assoc. 11, 111-121.

MacGregor, M.E. (1915) Notes on the rearing of Stegomyia fasciata in London. J. Trop. Med. (Hyg.) 18, 193-196.

Michalakis, Y. and Hochberg, M.E. (1994) Parasitic effects on host life-history traits: a review of recent studies. Parasite 1, 291-294.

Michalakis, Y., Olivieri, I., Renaud, F. and Raymond, M. (1992) Pleiotropic action of parasites: how to be good for the host. Trends Evol. Ecol. 7, 59-62.

Müller, C.B. and Schmid-Hempel, P. (1993) Exploitation of cold temperature as defense against parasitoids in bumbleebees. Nature 363, 65-67.

Philippi, T. and Seger, J. (1989) Hedging one's evolutionary bets, revisited. Trends Evol. Ecol. 4, $41-44$.

SAS Institute. (1994) JMP. Cary: SAS Institute Inc.

Seger, J. and Brockman, H.J. (1987) What is bet-hedging? In P. Harvey and L. Partridge (ed.) Oxford Surveys of Evolutionary Biology, vol. 4, pp. 182-211. Oxford University Press, Oxford.

Sokal, R.R. and Rohlf, F.J. (1995) Biometry, the principles and practice of statistics in biological research. W. H. Freeman and Company, San Fransisco.

Southwood, T.R.E., Murdie, G., Yasuno, M., Tonn, R.J. and Reader, P.M. (1972) Studies on the life budget of Aedes aegypti in Wat Samphaya, Bangkok, Thailand. Bull. Wld. Hlth. Org. 46, 211-226.

Undeen, A.H. and Becnel, J.J. (1992) Longevity and germination of Edhazardia aedis (Microspora, Amblyosporidae) spores. Biocontrol Sci. Technol. 2, 247-256. 